\title{
Descripción del perfil psicopatológico y delictivo en pacientes drogodependientes en tratamiento
}

\author{
José L. Rodríguez-Sáez ${ }^{1,2}$ y Alfonso Salgado-Ruiz ${ }^{3}$ \\ ${ }^{1}$ Fundación Aldaba - Proyecto Hombre, Valladolid, España \\ ${ }^{2}$ Universidad de Valladolid, Valladolid, España \\ ${ }^{3}$ Universidad Pontificia de Salamanca, Salamanca, España
}

\begin{abstract}
Description of the psychopathological and criminal profile in drug-dependent patients under treatment. This study performs an analysis of the prevalence of criminal behaviors in addicted patients under treatment. The sample consisted of 71 addicts (63 men and 8 women) who sought treatment at Proyecto Hombre Valladolid. Criminal behaviors, socio-demographic characteristics and consumption variables were evaluated with the EuropASI; psychopathological symptomatology with the SCL-90-R; and personality variables with the MCMI-III. Patients with and without criminal behaviors were compared in all the variables studied. The rate of addicted patients involved in criminal acts was $67.6 \%(\mathrm{n}=48)$. Significant differences were observed between patients with and without criminal behavior. Patients with criminal acts were more likely to show polydrug use. Patients with associated criminal behaviors had a more severe addiction. The implications of these results for clinical practice and future research are discussed.
\end{abstract}

Keywords: Drug addiction; crime; criminological profile; comorbidity; addictions.

Resumen: En este estudio se lleva a cabo un análisis de la prevalencia de conductas delictivas en pacientes adictos en tratamiento. Se cuenta con una muestra de 71 adictos (63 hombres y 8 mujeres) que acudieron a tratamiento en Proyecto Hombre Valladolid. Se evaluaron las conductas delictivas, las características sociodemográficas y las variables de consumo con el EuropASI, la sintomatología psicopatológica con el SCL-90-R y las variables de personalidad con el MCMI-III. Se compararon los pacientes con y sin conductas delictivas en todas las variables estudiadas. La tasa de pacientes adictos implicados en actos delictivos fue del $67.6 \%(n=48)$. Se observaron diferencias significativas entre los pacientes con y sin conductas delictivas. Entre los pacientes con actos delictivos era más probable que presentaran policonsumo de sustancias. Los pacientes con conductas delictivas asociadas presentaban una mayor gravedad en su adicción. Se discuten las implicaciones de estos resultados para la práctica clínica y la investigación futura.

Palabras clave: Drogadicción; delito; perfil criminológico; comorbilidad; adicciones.

\section{Introducción}

La experiencia clínica evidencia que muchas personas que acuden a tratamiento por problemas de drogas presentan también importantes problemas en otras áreas de su vida como salud física y mental, empleo, relaciones sociofamiliares, etc. (Ana et al., 2008; Fernán-

Recibido: 10 de febrero 2019; aceptado: 2 de octubre 2019

Correspondencia: Dr. José Luis Rodríguez Sáez, Facultad de Educación y Trabajo Social. Universidad de Valladolid. Paseo de Belén, 1 - 47011 Valladolid. España. Correo-e: joseluis.rodriguez@uva.es
dez-Montalvo, López-Goñi., Arteaga y Cacho, 2013). Aunque el consumo de drogas no conlleve de forma irremediable el implicarse en comportamientos delictivos, sin embargo, hay sujetos en que el abuso de sustancias psicoactivas está relacionado directamente con la actividad delictiva irremediablemente (Esbec y Echeburúa, 2016; Fernández-Montalvo, López-Goñi, y Arteaga, 2011, 2012). Varios estudios han encontrado que, en presos de todo el mundo, entre el $18-30 \%$ de los hombres y entre el $10-24 \%$ de las mujeres sufren de abuso o dependencia de alcohol y entre el $10-48 \%$ de hombres y entre el $30-60 \%$ de mujeres abusan o son dependientes de 
otras drogas (Fazel, Bains, y Doll, 2006). La media de población reclusa en España en el año 2016 fue de más de 52307 internos (Ministerio del Interior, 2017), y según la Encuesta sobre salud y consumo de drogas en internados en instituciones penitenciaria (ESDIP, 2016) de ese mismo año, el 70.8\% de los internos había consumido alguna droga ilegal en su vida, reduciéndose el porcentaje de consumidores en prisión en los últimos 30 días al 20.7\%. Y según el informe de prevalencia de trastornos mentales en prisiones españolas (Vicens et al., 2011), con una muestra de 783 varones, un $75 \%$ presentaba historia de trastorno por uso de sustancias, principalmente alcohol y cocaína.

Aunque diversos estudios señalan el nexo de relación entre drogas y delincuencia (Bennett y Holloway, 2005; Esbec y Echeburúa, 2016; Felson y Staff, 2010; Fernández-Montalvo et al., 2013; Menard, Mihalic, y Huizinga, 2001; Ramos y Garrote, 2009), la naturaleza y la dirección causal de dicha relación no ha sido identificada (Kuhns y Clodfelter, 2009; Ramos y Garrote, 2009). En otras palabras, pese a los datos proporcionados por diferentes estudios que ponen de relieve la relación drogas-delincuencia, estos resultados no permiten responder válidamente a la pregunta ¿qué causa qué? La literatura apunta tres hipótesis. La primera señala que la conducta delictiva se produce como consecuencia de la drogadicción del individuo. En este caso, la droga se vería como una necesidad, y el individuo que la consume se introduciría en el mundo delincuencial por la necesidad económica que se deriva de ese consumo. Esta hipótesis postula, pues, que la adicción puede conducir a comportamientos delictivos para financiar sus hábitos adictivos y evitar los síntomas de la abstinencia. La segunda hipótesis explicativa recogida en la literatura científica postula que la actividad delictiva precede el consumo de drogas, y el uso de sustancias ocurre en el contexto de un estilo de vida criminal y de conductas antisociales (Bennett y Holloway, 2005; Ramos y Garrote, 2009). Esta segunda explicación parte del supuesto de que el consumo de drogas es una fase avanzada dentro del historial delictivo de los individuos inmersos en subculturas «desviadas»: el sujeto no es primariamente adicto aunque más adelante termine recalando en el submundo de la droga por su propia inestabilidad personal o por la propensión a vulnerar las normas. Esto es, considera que es precisamente la implicación en la delincuencia lo que proporciona al sujeto el contexto, el grupo de referencia y las definiciones favorables de la situación que le conducirán a su posterior implicación en el consumo. Por tanto, la adicción a la droga no es más que una prolongación de la conducta desviada. Esta segunda hipó- tesis tiene un mayor apoyo empírico, si bien la práctica totalidad de los estudios no examinan la relación en términos de causalidad sino en términos de cuál es la conducta que ocurre primero, lo que es criterio necesario pero no suficiente para demostrar relación causal (Otero-López, 1997, 1999). La tercera hipótesis es que no existe relación causal entre ambas conductas. La relación entre droga y delito podría ser artificiosa y obedecer a un factor latente e inobservado que subyace en ambos comportamientos, como pueden ser situaciones de vulnerabilidad familiar o de exclusión social o un síndrome de comportamiento antisocial general (Contreras, Molina y Cano, 2012; Esbec y Echeburúa, 2016; Ramos y Garrote, 2009; Santo-Domingo, 2002; Valenzuela y Larroulet, 2010).

Además, como señalan Fernández-Montalvo et al., (2013), aunque muchos estudios han investigado el abuso de sustancias en población penitenciaria (Fazel et al., 2006), muy pocos estudios han evaluado personas con convicciones delictivas que participaran en programas de tratamiento de drogas. Esta escasez de estudios es sorprendente porque se estima que el $38.1 \%$ de los pacientes tratados en programas comunitarios de tratamiento de drogas y el $26.2 \%$ de los pacientes tratados en programas residenciales participaban en conductas delictivas en el año anterior a la entrada (Gossop et al., 2005). Algunos estudios han abordado el problema de la conducta delictiva en adictos. Por ejemplo, un estudio reciente que compara los perfiles de pacientes tratados en una unidad de tratamiento penitenciaría y en una comunidad terapéutica destacó una mayor severidad de la adicción en los pacientes encarcelados (Casares-López et al., 2010). Cuestiones relacionadas con la situación jurídica de los pacientes también se han abordado con frecuencia en la investigación que evalúa diferentes programas de tratamiento (Fernández-Montalvo y López-Goñi, 2010; Fernández-Montalvo et al., 2008), en estudios de seguimiento de pacientes (Gossop et 2005), y en la investigación para identificar diferentes categorías de pacientes (Graña, Muñoz, y Navas, 2009). Todos estos estudios demostraron la existencia de una estrecha relación entre drogadicción y comportamiento criminal.

Sin embargo, como indican Fernández-Montalvo et al., (2013), poco se sabe sobre el perfil específico de los pacientes adictos con problemas de conducta delictiva o en qué medida estos individuos se diferencian de otros drogodependientes sin estos problemas. Conocer las características del primer grupo (variables sociodemográficas, síntomas psicopatológicos, rasgos de personalidad, etc.) es fundamental para adaptar los programas de tratamiento existentes a los problemas 
particulares de estos pacientes, como ha sido indicado en estudios previos sobre los perfiles diferenciales en pacientes drogodependientes (Graña et al., 2009; Fernández-Montalvo y López-Goñi, 2010; Fernández-Montalvo et al., 2012). Así, este estudio examinó la prevalencia del comportamiento criminal en una muestra de sujetos con trastorno por uso de sustancias que buscaron tratamiento en Proyecto Hombre Valladolid. Los principales objetivos de este estudio fueron identificar las diferentes características de los sujetos drogodependientes con problemas relacionados con conductas delictivas y determinar los perfiles específicos que diferencian a los pacientes con y sin delitos relacionados con problemas legales. La principal hipótesis era que los pacientes con comportamientos delictivos presentarían una adicción más severa.

\section{Método}

\section{Participantes}

La muestra de este estudio está compuesta por 71 pacientes que se encontraban al inicio del tratamiento de rehabilitación por adicción a diferentes sustancias en Proyecto Hombre de Valladolid. Se excluyeron de la muestra los sujetos que presentaran alteraciones psicóticas graves, abandonaran el tratamiento antes de poder ser evaluados o fueran incapaces de responder a los instrumentos de evaluación utilizados. Y con el fin de evitar la posible influencia de síntomas de deprivación o intoxicación se consideró, como criterio de inclusión, presentar un período de entre tres y seis semanas de abstinencia de drogas en el momento de la evaluación.

La edad media de los individuos incluidos en el estudio fue 32.2 años $(S D=7.4)$; la muestra incluyó 63 $(88.7 \%)$ hombres y $8(11.3 \%)$ mujeres. El nivel socioeconómico fue entre medio y medio-bajo. Las principales sustancias por las que demandaron tratamiento fueron el alcohol, de forma sola o conjuntamente con otras drogas, (29.6\% de la muestra); la cocaína (23.9\%); el policonsumo (22.6\%); y el cannabis (15.5\%) seguido por otras sustancias (por ejemplo, anfetaminas, heroína, etc.) en menor número ( $8.4 \%$ de la muestra).

\section{Instrumentos}

EuropASI (Kokkevi y Hartgers, 1995; versión española de Bobes et al., 1996). Es la versión europea del Índice de Severidad de la Adicción (ASI) (McLellan et al., 1980). Se trata de una entrevista clínica semiestructurada y heteroaplicada de 159 ítems que evalúan la gravedad de la adicción y otras seis áreas que han podido contribuir al inicio y mantenimiento de la adicción: estado médico general, situación laboral y financiera, consumo de alcohol, consumo de otras drogas, problemas legales, relaciones familiares y sociales y, por último, estado psicológico. En este estudio se utilizaron los ítems de la escala legal para obtener información específica sobre la presencia de problemas legales. Para la escala de situación legal, la consistencia interna (alpha de Cronbach) tenía una puntuación de .504 para este estudio. En este sentido, se ha registrado el número y tipo de cargos formales acumulados por el paciente durante su vida, aunque no todos han supuesto necesariamente detención, interrogatorio, sanción o condena.

Symptom Checklist-90-R (SCL-90-R; Derogatis, 1992; versión en español de González de Rivera, 2002). Es uno de los instrumentos más utilizados para el autoinforme de síntomas. Procede de la revisión de la versión inicial, el SCL-90 (Symptom Check List-90), desarrollada por Derogatis y colaboradores en 1973, cuyo origen surge del listado de síntomas de la Universidad de Johns Hopkins HSCL en los años 50. Se trata de un cuestionario breve y multidimensional, autoinformado, de 90 ítems. Las respuestas se presentan en una escala tipo Likert (0-4), en función de la intensidad con la que el sujeto ha vivido en las últimas semanas el malestar que cada ítem explora. Está diseñado como instrumento de exploración de problemas psicológicos y de síntomas psicopatológicos en adolescentes y adultos. Se observó una elevada consistencia interna en las nueve subescalas del instrumento, a excepción de las susbescala de síntomas paranoides, que tuvo una puntuación de .66. El resto de las escalas oscilaron con un alpha de Cronbach de .78 a .88 .

Inventario Clínico Multiaxial de Millon (MCMI-III; Millon, Davis y Millon, 1997; adaptación española de Cardenal y Sánchez, 2007): Es un cuestionario clínico autoaplicado que posibilita la evaluación tanto de la personalidad del sujeto como de distintos síndromes clínicos. Está compuesto por 175 ítems de respuesta dicotómica (verdadero/falso), que proporciona información sobre once patrones clínicos de personalidad, tres rasgos patológicos, siete síndromes de gravedad moderada y otros tres de gravedad severa. En nuestra muestra de 71 sujetos, la consistencia interna obtenida es muy similar a la validación del cuestionario. Se obtienen coeficientes de fiabilidad superiores a .75 también en 21 escalas.

\section{Procedimiento}

La muestra seleccionada se extrajo a partir de los ingresos realizados durante el período comprendido entre 
enero de 2013 a marzo de 2014. La evaluación se llevó a cabo en el marco de la evaluación pretratamiento. Aquellos pacientes que cumplían los criterios de admisión y que aceptaron formar parte de la investigación, fueron citados para ser evaluados en dos sesiones, siguiendo criterios del DSM5. En la primera se recogieron los datos relacionados con las características sociodemográficas y de consumo de sustancias. En la segunda sesión se evaluó la presencia de sintomatología psicopatológica y trastornos de personalidad con el SCL-90-R y el MCMI-III. Después de las sesiones de evaluación, los pacientes iniciaron el tratamiento estándar en Proyecto Hombre para la adicción.

\section{Análisis estadístico}

Se realizaron análisis descriptivos para todas las variables. Los análisis bivariados se emplearon utilizando $\chi^{2}$ o la prueba $U$ de Mann-Whitney, dependiendo de la naturaleza de las variables estudiadas. Una diferencia de $p<.05$ fue considerada significativa. Los análisis estadísticos fueron realizados con el programa SPSS v. 19. En todos los casos se consideró $\alpha<.05$.

\section{Resultados}

\section{Perfiles criminológicos de los pacientes con adicción en tratamiento}

En la muestra, el $67.6 \%$ de los adictos habían sido acusados formalmente de un delito. El número fue ma- yor para hombres que para mujeres; el $69.8 \%$ de los pacientes varones habían sido acusado de un delito en comparación con el 50\% de las mujeres (Véase la Tabla 1).

Un análisis más detallado del tipo de delito reveló que la mayoría estaban relacionados con delitos contra la seguridad vial y robos (p.ej. robos en domicilios, tiendas, fraudes). De la muestra total, el $32.4 \%$ de los pacientes (30.2\% de los varones y $50 \%$ de las mujeres) había sido acusado de un delito contra el patrimonio; sin embargo, las diferencias de género encontradas para esta categoría de delitos no fueron estadísticamente significativas. Para el resto de delitos no se pudo establecer comparaciones ya que las mujeres solamente habían sido acusadas de delitos contra el patrimonio. En los delitos contra la seguridad vial (p.ej. conducción temeraria, exceso de velocidad, conducción alcohólica, etc.), el segundo tipo de delito más frecuente, estaban involucrados el $33.8 \%$ de la muestra (38.1\% de los varones). En los delitos contra la salud pública (CSP) estaban implicados el $18.3 \%$ de la muestra (20.6\% de los varones). Los delitos menos frecuentes fueron los relacionados con la trata de personas, alteración del orden, delitos violentos y otros tales como la evasión fiscal o el impago de la pensión a la expareja o hijos. Además, el 31\% de la muestra total había cumplido condena anteriormente, y el $23.2 \%$ resultó ser una pena de prisión, el $23.9 \%$ estaban a la espera de cargos, juicio o sentencia, y el 1.4\% había sido previamente condenado por un delito y estaba en libertad condicional.

Tabla 1. Características criminológicas de los usuarios

\begin{tabular}{|c|c|c|c|}
\hline & $\begin{array}{c}\text { Todos } \\
(N=71) \\
n(\%)\end{array}$ & $\begin{array}{c}\text { Hombres } \\
(n=63) \\
n(\%)\end{array}$ & $\begin{array}{c}\text { Mujeres } \\
(n=8) \\
n(\%)\end{array}$ \\
\hline Acusados de un delito & $48(67.6 \%)$ & $44(69,8 \%)$ & $4(50 \%)$ \\
\hline Delitos contra el patrimonio & $23(32.4 \%)$ & $19(30,2 \%)$ & $4(50 \%)$ \\
\hline Delitos contra la seguridad vial & $24(33.8 \%)$ & $24(38,1 \%)$ & $0(0 \%)$ \\
\hline Delitos contra la salud pública & $13(18.3 \%)$ & $13(20,6 \%)$ & $0(0 \%)$ \\
\hline Otros delitos & $10(14.1 \%)$ & $10(15,9 \%)$ & $0(0 \%)$ \\
\hline Delitos violentos & $9(12.7 \%)$ & $9(14,3 \%)$ & $0(0 \%)$ \\
\hline Alteración del orden & $7(9.9 \%)$ & $7(11,1 \%)$ & $0(0 \%)$ \\
\hline Proxenetismo & $1(1.4 \%)$ & $1(1,6 \%)$ & $0(0 \%)$ \\
\hline Condenados & $22(31 \%)$ & $21(33.3 \%)$ & $1(12.5 \%)$ \\
\hline Encarcelados & $16(23.2 \%)$ & $16(26.2 \%)$ & $0(0 \%)$ \\
\hline Pendientes de cargos, juicio o sentencia & $17(23.9 \%)$ & $15(23.8 \%)$ & $2(25 \%)$ \\
\hline En libertad condicional & $1(1.4 \%)$ & $1(1.6 \%)$ & $0(0 \%)$ \\
\hline
\end{tabular}


Comparaciones de pacientes adictos con y sin comportamiento delictivo

Variables sociodemográficas y de consumo de drogas. Con respecto a las variables sociodemográficas, no se observaron diferencias significativas entre los pacientes que habían sido acusados de cometer delitos y los que no tenían cargos formales (ver Tabla 2). En particular, el $91.7 \%$ de los pacientes que habían participado en conductas delictivas eran hombres, mientras que el $82.6 \%$ de los pacientes sin problemas legales eran varones.
Sin embargo, sí se observaron diferencias en el tipo de abuso de sustancias que condujeron a buscar tratamiento entre los pacientes que habían tenido o no problemas legales (Tabla 2). Así, el grupo con problemas legales exhibió significativamente mayor policonsumo en comparación con aquellos que no habían sido acusados de participar en conductas delictivas. Aunque el porcentaje de pacientes con sobredosis fue mayor en el grupo de pacientes con problemas relacionados con el comportamiento delictivo, esta diferencia no fue estadísticamente significativa.

Tabla 2 Comparación en variables sociodemográficas y de consumo

\begin{tabular}{|c|c|c|c|c|c|c|c|}
\hline & \multicolumn{2}{|c|}{$\begin{array}{c}\text { Todos } \\
(N=71)\end{array}$} & \multicolumn{2}{|c|}{$\begin{array}{l}\text { Acusados de un delito } \\
(n=48)\end{array}$} & \multicolumn{2}{|c|}{$\begin{array}{l}\text { No acusados de un delito } \\
\qquad(n=23)\end{array}$} & \multirow[b]{2}{*}{$t$} \\
\hline & Media & $(S D)$ & Media & $(S D)$ & Media & $(S D)$ & \\
\hline Edad & 32.24 & 7.444 & 32.38 & 6.819 & 31.96 & 8.767 & -.202 \\
\hline Sexo & $N$ & $(\%)$ & $n$ & $(\%)$ & $n$ & $(\%)$ & $X^{2}(g l)$ \\
\hline Varón & 63 & $88.7 \%$ & 44 & $91.7 \%$ & 19 & $82.6 \%$ & \multirow{2}{*}{$1.276(1)$} \\
\hline Mujer & 8 & $11.3 \%$ & 4 & $8.3 \%$ & 4 & $17.4 \%$ & \\
\hline Estado civil & $N$ & $(\%)$ & $n$ & $(\%)$ & $n$ & $(\%)$ & $X^{2}(g l)$ \\
\hline Casado & 10 & $14.1 \%$ & 6 & $12.5 \%$ & 4 & $17.4 \%$ & \multirow{3}{*}{$1.558(3)$} \\
\hline Separado/divorciado & 12 & $16.9 \%$ & 8 & $16.7 \%$ & 4 & $17.4 \%$ & \\
\hline Soltero & 49 & $69 \%$ & 34 & $70.8 \%$ & 15 & $65.2 \%$ & \\
\hline Grado académico & $N$ & (\%) & $n$ & $(\%)$ & $n$ & $(\%)$ & $X^{2}(g l)$ \\
\hline Sin estudios & 3 & $4.2 \%$ & 1 & $2.1 \%$ & 2 & $8.7 \%$ & \\
\hline Primaria & 39 & $54.9 \%$ & 27 & $56.3 \%$ & 12 & $52.2 \%$ & \multirow{3}{*}{$3.930(3)$} \\
\hline Secundaria/FP & 28 & $39.4 \%$ & 20 & $41.7 \%$ & 8 & $34.8 \%$ & \\
\hline Universitarios/Grado & 1 & $1.4 \%$ & 0 & $0 \%$ & 1 & $4.3 \%$ & \\
\hline Situación laboral & $N$ & $(\%)$ & $n$ & (\%) & $n$ & $(\%)$ & \multirow[t]{2}{*}{$X^{2}(g l)$} \\
\hline Empleado & 44 & $62 \%$ & 29 & $60.4 \%$ & 15 & $65.2 \%$ & \\
\hline Desempleado & 16 & $22.5 \%$ & 10 & $20.8 \%$ & 6 & $26.1 \%$ & \multirow[t]{2}{*}{$1.263(2)$} \\
\hline Otros (estudiante, retirado...) & 11 & $15.5 \%$ & 9 & $18.8 \%$ & 2 & $8.7 \%$ & \\
\hline Sustancia que motiva el tratamiento & $N$ & $(\%)$ & $n$ & $(\%)$ & $n$ & $(\%)$ & \multirow[t]{2}{*}{$X^{2}(g l)$} \\
\hline Depresoras (alcohol, heroína, cannabis) & 15 & $21.1 \%$ & 10 & $20.8 \%$ & 5 & $21.7 \%$ & \\
\hline Estimulantes (cocaína y anfetaminas) & 21 & $29.6 \%$ & 12 & $25 \%$ & 9 & $39.1 \%$ & \multirow{3}{*}{$10.668(3)^{*}$} \\
\hline Alcohol y otras drogas & 19 & $26.8 \%$ & 10 & $20.8 \%$ & 9 & $39.1 \%$ & \\
\hline Policonsumo & 16 & $22.5 \%$ & 16 & $33.3 \%$ & 0 & $0 \%$ & \\
\hline Sobredosis & $N$ & $(\%)$ & $n$ & $(\%)$ & $n$ & $(\%)$ & $X^{2}(g l)$ \\
\hline Sí & 18 & $25.4 \%$ & 14 & $29.2 \%$ & 4 & $17.4 \%$ & \multirow{2}{*}{$1.139(1)$} \\
\hline No & 53 & $74.6 \%$ & 34 & $70.8 \%$ & 19 & $82.6 \%$ & \\
\hline
\end{tabular}

Nota: ${ }^{*} p<.05$

Las puntuaciones del Índice Europeo de Severidad de la Adicción (EuropASI) revelaron diferencias significativas entre los grupos con respecto a la gravedad de la adicción (Tabla 3). Los pacientes adictos que tuvieron problemas legales tuvieron puntuaciones significativa- mente más altas que los individuos sin problemas legales y una mayor necesidad de tratamiento en las áreas de asuntos legales y apoyo familiar. Y se observa una diferencia que tiende a ser significativa en las áreas médica y social. 
Tabla 3. Comparación en puntuaciones de severidad del EuropASI

\begin{tabular}{lccc}
\hline & \multicolumn{1}{c}{ Acusados de un delito $(n=48)$} & No acusados de un delito $(n=23)$ & \\
\cline { 2 - 3 } \multicolumn{1}{c}{ EuropASI } & Rango medio & Rango medio & U de Mann-Whitney \\
\hline Médica & 38.30 & 31.20 & 441.5 \\
Empleo/Soporte & 30.89 & 35.37 & 405.5 \\
Uso de Alcohol & 34.49 & 39.15 & 479.5 \\
Uso de Drogas & 36.67 & 34.61 & 520 \\
Legal & 42.35 & 22.74 & $247^{* * *}$ \\
Familiar & 40.54 & 26.52 & $334^{* *}$ \\
Social & 38.96 & 29.83 & 410 \\
Psiquiátrica & 36.56 & 34.83 & 525 \\
\hline
\end{tabular}

Nota: $* * * p<.001 ; * * \mathrm{p}<.01$

Variables psicopatológicas y de la personalidad. Las puntuaciones de los pacientes en el (SCL-90-R) indican un nivel moderado a alto de síntomas psicopatológicos en la muestra del estudio (ver Tabla 4). A pesar de que los pacientes con trastorno por uso de sustancias con problemas legales obtuvieron puntuaciones medias mayores en todas las escalas del SCL-90-R sin embargo, no hubo diferencias estadísticamente significativas entre los individuos que tenían problemas asociados con el comportamiento delictivo y los que no habían sido acusados de delitos; aunque se observó una tendencia a alcanzar diferencias significativas para las escalas de Somatiza- ción, Obsesión-compulsión, Sensibilidad interpersonal, Ansiedad, Psicoticismo, GSI y PST.

Con respecto a los rasgos de personalidad, las puntuaciones de los pacientes en el MCMI-III revelaron la existencia de diferencias significativas en los dos grupos de pacientes tan solo para la escala Paranoide de la personalidad (Tabla 5); mientras que los individuos que nunca habían sido acusados de un delito obtuvieron una puntuación más alta en la escala de trastorno de la personalidad narcisista con una tendencia a alcanzar significación estadística.

Tabla 4. Comparación en las variables del SCL-90-R

\begin{tabular}{lccccc}
\hline & \multicolumn{2}{c}{ Acusados de delito $(n=48)$} & \multicolumn{2}{c}{ No acusados de delito $(n=23)$} & \\
\cline { 2 - 4 } \multicolumn{1}{c}{ SCL-90-R } & Media $(D T)$ & Rango promedio & Media $(D T)$ & Rango promedio & U de Mann-Whitney \\
\hline GSI & $1.33(0.80)$ & 38.83 & $1.04(0.76)$ & 30.09 & 416.0 \\
PSDI & $1.99(0.64)$ & 38.34 & $1.80(0.59)$ & 31.11 & 439.5 \\
PST & $56.56(19.37)$ & 38.60 & $47.61(23.79)$ & 30.57 & 427.0 \\
Somatización & $1.14(0.84)$ & 38.84 & $0.86(0.83)$ & 30.07 & 415.5 \\
Obsesión-compulsión & $1.46(0.90)$ & 38.57 & $1.12(0.81)$ & 30.63 & 428.5 \\
Sensibilidad interp. & $1.51(0.97)$ & 38.66 & $1.15(0.94)$ & 30.46 & 424.5 \\
Depresión & $1.66(0.92)$ & 37.93 & $1.45(1.05)$ & 31.98 & 459.5 \\
Ansiedad & $1.33(0.92)$ & 38.79 & $0.96(0.83)$ & 30.17 & 418 \\
Hostilidad & $0.99(0.92)$ & 37.17 & $0.86(0.97)$ & 33.57 & 496 \\
Ansiedad fóbica & $0.87(0.91)$ & 37.29 & $0.60(0.56)$ & 33.30 & 490 \\
Ideación Paranoide & $1.44(0.88)$ & 38.34 & $1.19(0.92)$ & 31.11 & 439.5 \\
Psicoticismo & $1.20(0.90)$ & 38.63 & $0.86(0.76)$ & 30.52 & 426 \\
\hline
\end{tabular}

Nota: GSI (Índice sintomático general); PSDI (Índice de distrés de síntomas positivos) y el PST (Total de síntomas positivos). 
Tabla 5. Comparación en las escalas de personalidad del MCMI-III

\begin{tabular}{|c|c|c|c|c|c|}
\hline \multirow[b]{2}{*}{ MCMI-III } & \multicolumn{2}{|c|}{ Acusados de un delito $(n=48)$} & \multicolumn{2}{|c|}{ No acusados de un delito $(n=23)$} & \multirow[b]{2}{*}{$U$ de Mann-Whitney } \\
\hline & Media $(D T)$ & Rango promedio & Media $(D T)$ & Rango promedio & \\
\hline Esquizoide & $50.3(22.0)$ & 35.96 & $46.4(24.8)$ & 36.09 & 550 \\
\hline Evitativa & $55.8(18.8)$ & 37.47 & $47.3(27.2)$ & 32.93 & 481.5 \\
\hline Depresiva & $55.2(21.8)$ & 37.38 & $46.3(26.6)$ & 33.13 & 486 \\
\hline Dependiente & $50.4(21.2)$ & 37.46 & $45.4(22.1)$ & 32.96 & 482 \\
\hline Histriónica & $30.9(18.3)$ & 34.39 & $38.9(25.5)$ & 39.37 & 474.5 \\
\hline Narcisista & $56.5(15.5)$ & 33.16 & $60.6(15.9)$ & 41.93 & 415.5 \\
\hline Antisocial & $70.3(14.5)$ & 37.26 & $68.4(12.7)$ & 33.37 & 491.5 \\
\hline Agresiva (sádica) & $13.4(5.4)$ & 37.03 & $12.3(6.0)$ & 33.85 & 502.5 \\
\hline Compulsiva & $37.0(18.3)$ & 34.85 & $38.0(17.0)$ & 38.39 & 497 \\
\hline Negativista & $54.5(14.7)$ & 37.28 & $49.2(20.1)$ & 33.33 & 490.5 \\
\hline Autodestructiva & $48.2(17.7)$ & 37.97 & $37.9(24.5)$ & 31.89 & 457.5 \\
\hline Esquizotípica & $55.0(19.2)$ & 37.93 & $44.7(28.1)$ & 31.98 & 459.5 \\
\hline Límite & $59.0(14.3)$ & 37.93 & $51.5(18.0)$ & 31.98 & 459.5 \\
\hline Paranoide & $60.8(18.7)$ & 39.52 & $46.4(27.3)$ & 28.65 & $383^{*}$ \\
\hline
\end{tabular}

Nota: $* p<.05$.

En cuanto a los trastornos de la personalidad, el 50\% de los pacientes $(n=24)$ presentaron al menos un trastorno de la personalidad (ver Tabla 6). Sin embargo, los grupos con y sin problemas relacionados con la delincuencia no difieren en la tasa global de trastornos de la personalidad, salvo en la escala de trastorno de la personalidad histriónica. Los trastornos de personalidad antisocial, paranoide y evitativa fueron los más prevalentes entre el grupo con problemas relacionados con la delincuencia; mientras que aquellos que nunca habían sido acusados de un delito exhibieron una tasa más alta de trastorno de personalidad antisocial, histriónica y depresiva.

Variables de desajuste. En la Tabla 7 se presentan los resultados de la comparación entre grupos de pacientes para varias variables de desajuste. Todos los pacientes presentaron altas tasas de problemas con diferentes miembros de la familia, sin diferencias significativas entre los dos grupos salvo para la variable problemas con vecinos.

Tabla 6. Comparación en la tasa de trastornos de la personalidad (PREV>74)

\begin{tabular}{|c|c|c|c|c|c|c|c|}
\hline & \multicolumn{2}{|c|}{ Todos $(N=71)$} & \multicolumn{2}{|c|}{ Acusados de un delito $(n=48)$} & \multicolumn{2}{|c|}{ No acusados de un delito ( $n=23)$} & \multirow[b]{2}{*}{ Fisher (sig) } \\
\hline & $\mathrm{N}$ & $(\%)$ & $\mathrm{n}$ & $(\%)$ & $\mathrm{n}$ & $(\%)$ & \\
\hline Esquizoide & 3 & $4.2 \%$ & 2 & $4.2 \%$ & 1 & $4.3 \%$ & 1.000 \\
\hline Evitativa & 7 & $9.9 \%$ & 6 & $12.5 \%$ & 1 & $4.3 \%$ & .415 \\
\hline Depresiva & 7 & $9.9 \%$ & 5 & $10.4 \%$ & 2 & $8.7 \%$ & 1.000 \\
\hline Dependiente & 4 & $5.6 \%$ & 3 & $6.3 \%$ & 1 & $4.3 \%$ & 1.000 \\
\hline Histriónica & 4 & $5.6 \%$ & 0 & $0 \%$ & 4 & $17.4 \%$ & .009 \\
\hline Narcisista & 8 & $11.3 \%$ & 5 & $10.4 \%$ & 3 & $13 \%$ & .708 \\
\hline Antisocial & 21 & $29.6 \%$ & 16 & $33.3 \%$ & 5 & $21.7 \%$ & .409 \\
\hline Agresiva (sádica) & 1 & $1.4 \%$ & 0 & $0 \%$ & 1 & $4.3 \%$ & .324 \\
\hline Compulsiva & 2 & $2.8 \%$ & 2 & $4.2 \%$ & 0 & $0 \%$ & 1.000 \\
\hline Negativista & 0 & $0 \%$ & 0 & $0 \%$ & 0 & $0 \%$ & \\
\hline Autodestructiva & 0 & $0 \%$ & 0 & $0 \%$ & 0 & $0 \%$ & \\
\hline Esquizotípica & 2 & $2.8 \%$ & 1 & $2.1 \%$ & 1 & $4.3 \%$ & .546 \\
\hline Límite & 1 & $1.4 \%$ & 0 & $0 \%$ & 1 & $4.3 \%$ & .324 \\
\hline Paranoide & 7 & $9.9 \%$ & 6 & $12.5 \%$ & 1 & $4.3 \%$ & .415 \\
\hline Total $^{\mathrm{a}}$ & 37 & $52.1 \%$ & 24 & $50 \%$ & 13 & $56.5 \%$ & .623 \\
\hline
\end{tabular}

Nota: a El número total de personas afectadas por trastornos de la personalidad es inferior a la suma total de trastornos porque hay pacientes que presentan más de un trastorno de la personalidad. 
Tabla 7. Comparación en variables de desajuste familiar/social y abusos

\begin{tabular}{|c|c|c|c|c|c|c|c|c|c|}
\hline & & \multirow[b]{2}{*}{$N$} & \multicolumn{2}{|c|}{$\begin{array}{c}\text { Todos } \\
(N=71)\end{array}$} & \multicolumn{2}{|c|}{$\begin{array}{l}\text { Acusados de un delito } \\
\qquad(n=48)\end{array}$} & \multicolumn{2}{|c|}{$\begin{array}{l}\text { No acusados de un delito } \\
(n=23)\end{array}$} & \multirow[b]{2}{*}{ Fisher (sig.) } \\
\hline & & & $N$ & $(\%)$ & $n$ & $(\%)$ & $n$ & $(\%)$ & \\
\hline \multicolumn{10}{|c|}{ Desajuste familiar } \\
\hline \multirow{5}{*}{$\begin{array}{l}\text { problemas } \\
\text { con }\end{array}$} & Madre & 71 & 43 & $60.6 \%$ & 31 & $64.6 \%$ & 12 & $52.2 \%$ & .437 \\
\hline & Padre & 69 & 47 & $68.1 \%$ & 33 & $71.7 \%$ & 14 & $60.9 \%$ & .417 \\
\hline & Hermanos/as & 68 & 41 & $60.3 \%$ & 27 & $60 \%$ & 14 & $60.9 \%$ & 1.000 \\
\hline & Pareja & 64 & 44 & $68.8 \%$ & 32 & $74.4 \%$ & 12 & $57.1 \%$ & .250 \\
\hline & Hijos/as & 27 & 4 & $14.8 \%$ & 3 & $14.3 \%$ & 1 & $16.7 \%$ & 1.000 \\
\hline \multicolumn{10}{|c|}{ Desajuste social } \\
\hline \multirow{3}{*}{$\begin{array}{l}\text { problemas } \\
\text { con }\end{array}$} & Amigos & 69 & 26 & $37.7 \%$ & 17 & $36.2 \%$ & 9 & $40.9 \%$ & .792 \\
\hline & Vecinos & 71 & 16 & $22.5 \%$ & 14 & $29.2 \%$ & 2 & $8.7 \%$ & $.047^{*}$ \\
\hline & Compañeros trabajo & 69 & 13 & $18.8 \%$ & 10 & $20.8 \%$ & 3 & $14.3 \%$ & .740 \\
\hline \multicolumn{10}{|c|}{ Víctima de abuso } \\
\hline & Emocional/psicológico & 71 & 49 & $69 \%$ & 34 & $70.8 \%$ & 15 & $65.2 \%$ & .785 \\
\hline & Físico & 71 & 30 & $42.3 \%$ & 22 & $45.8 \%$ & 8 & $34.8 \%$ & .447 \\
\hline & Sexual & 71 & 5 & $7 \%$ & 4 & $8.3 \%$ & 1 & $4.3 \%$ & 1.000 \\
\hline
\end{tabular}

Nota: $* p<.05$.

Cabe señalar que no hubo diferencias significativas entre los grupos de pacientes respecto a haber sufrido abuso psicológico, físico o sexual (Tabla 7).

\section{Discusión}

Los resultados del estudio revelan que el comportamiento delictivo es frecuente entre los drogodependientes que demandan tratamiento en Proyecto Hombre Valladolid. De hecho, el $67.6 \%$ de la muestra del estudio refiere haber sido acusado por un comportamiento delictivo en un momento $u$ otro. La mayoría de los delitos estaban relacionados con robos, delitos contra la seguridad vial (p.ej. conducción temeraria, exceso de velocidad, conducción alcohólica,...), y delitos contra la salud pública. Este tipo de delitos suelen producirse durante la intoxicación (en el caso de los delitos relacionados con la conducción) o como un medio de satisfacerse los costes del consumo (en el caso de la venta de drogas). Los resultados del presente estudio sugieren que la fuerte conexión entre delincuencia y adicción podría estar relacionada con los efectos del consumo en el comportamiento, la necesidad urgente de obtener dinero para mantener el consumo de drogas y el comportamiento relacionado con el mercado ilegal de drogas. Estos resultados apuntan en la dirección de estudios previos (Fernández-Montalvo et al., 2013). De todos modos, esta hipótesis necesita investigación futura.

De acuerdo con Fernández-Montalvo et al. (2013), independientemente de la dirección causal de la relación, el elevado número de problemas legales asociados con la adicción que se encuentran en este estudio pone de relieve la necesidad de mejorar los procedimientos para recopilar información sobre el estatus legal de los pacientes con adicciones. Los datos de EuropASI proporcionan una medida global de las cuestiones jurídicas del paciente, pero sólo producen información limitada. Por otra parte, las escalas legales de la ASI y del EuropASI fueron las escalas menos fiables, tanto en lo que se refiere a los índices de Severidad del entrevistador como a los puntajes compuestos del instrumento (López-Goñi, Fernández-Montalvo y Arteaga, 2012). Por lo tanto, los protocolos de prueba diseñados para evaluar los problemas legales en los pacientes con adicciones deben ser mejorados. En este sentido, debe apuntarse que algunas referencias a comportamientos delictivos deben ser revisadas en la edición española del instrumento, porque no se ajustan adecuadamente a la actual legislación penal española.

El presente estudio no encontró diferencias significativas en función del sexo en el alcance de los problemas legales relacionados con el delito. Este hallazgo se aleja de los resultados de otros estudios que han hallado perfi- 
les diferenciales en cuanto a la variable sexo (Fernández-Montalvo et al., 2013; Lee, 2007; Tournier et al., 2005). Es preciso señalar que los resultados de este estudio se obtuvieron de una muestra que incluía pocas mujeres, por lo que es preciso una confirmación de estos resultados con muestras que incluyan un porcentaje mayor de mujeres.

Además, un hallazgo importante de este estudio es que los pacientes adictos con problemas legales exhibieron significativamente más policonsumo que los pacientes sin problemas legales. Estos resultados son consistentes con los de otros estudios (Fernández-Montalvo et al., 2013; Bennett y Holloway, 2005) y sugieren un tema prometedor para la investigación futura. La investigación existente sobre la asociación entre la adicción y el comportamiento delictivo se ha centrado principalmente en el análisis de la asociación de determinadas drogas con ciertos delitos, como los estudios sobre la relación entre el consumo de heroína y el delito. Sin embargo, hay poca investigación que analiza la relación entre el abuso de varias sustancias y la conducta criminal. Cuando se ha desarrollado este tipo de investigación, la conclusión más plausible parece ser que el comportamiento ilícito está influenciado por el uso de sustancias, en particular el uso ilícito de sustancias, y que el consumo de sustancias, en particular el uso de drogas ilícitas, está influenciado por el comportamiento ilegal (Menard et al., 2001).

La comparación entre los dos grupos, sin embargo, no reveló que los pacientes con problemas legales tuvieran adicciones más severas, como se ha encontrado en otros estudios (Fernández-Montalvo et al., 2013). Las puntuaciones del EuropASI, que miden la gravedad de la adicción, fueron significativamente mayores para las escalas relacionadas con cuestiones legales y problemas familiares, pero no para la escala relacionada con el alcohol y otras drogas.

Desde una perspectiva psicopatológica, el SCL-90-R no encontró diferencias entre los grupos en la sintomatología psicopatológica, si bien estuvieron próximas a la significación en las escalas de Somatización, Obsesión-compulsión, Sensibilidad interpersonal, Ansiedad, Psicoticismo, GSI y PST. En un estudio sobre la prevalencia de conductas delictivas en personas adictas en tratamiento con 252 participantes, Fernández-Montalvo et al., (2013) tampoco hallaron diferencias en sintomatología psicopatológica. El $50 \%$ de los pacientes $(\mathrm{n}=24)$ presentaron al menos un trastorno de la personalidad. Estos resultados confirman los hallados en un estudio anterior (Fernández-Montalvo et al., 2012) que compararon 252 pacientes adictos con y sin problemas relacionados con la violencia y encontraron que el $46.8 \%$ de los pacientes presentaban al menos un trastorno de personalidad. En cuanto a las escalas de personalidad del MCMI-III, se revelaron algunas diferencias. Así, los pacientes con problemas legales tuvieron puntuaciones significativamente más altas en la escala de trastorno de personalidad paranoide. No se encontraron diferencias entres los grupos para el trastorno antisocial de la personalidad como sucede en otros estudios similares (Fernández-Montalvo et al., 2012, 2013). Estos resultados inconsistentes con otros estudios para la escala antisocial podrían atribuirse a la posibilidad de una elevada deseabilidad de las personas encuestadas por los profesionales que los van a admitir a tratamiento, por lo que cabe esperar que se muestren preferentemente perfiles aplanados de personalidad en la mayoría de las escalas.

Los trastornos de personalidad antisocial, paranoide y evitativa fueron los más prevalentes entre el grupo con problemas relacionados con la delincuencia. Como era de esperar, los pacientes adictos con problemas legales relacionados con el delito obtuvieron una tasa de prevalencia más alta, aunque sin alcanzar significación estadística, en la escala de personalidad antisocial, lo cual coincide con el perfil específico de este grupo y es consistente con otras investigaciones sobre la relación de los trastornos de la personalidad con comportamientos adictivos (Fernández-Montalvo, Landa, López-Goñi y Lorea, 2006; Fernández-Montalvo, Lorea, López-Goñi, Landa y Zarzuela, 2003). En el caso del trastorno de personalidad paranoide, la frecuencia es esperable dadas las características que configuran el diagnóstico de este trastorno según el DSM-5: sospechas infundadas hacia los demás, preocupación con dudas no justificadas sobre la lealtad de amigos y socios, reticencia a confiar en otras personas por temor a que usen en su contra la información que comparta, extracción de significados ocultos en situaciones neutrales, rencor persistente, celos, percepción de ataques a su carácter o reputación que son inapreciables por los demás y rapidez para contraatacar en respuesta a las amenazas que perciben a su alrededor. Caldero (2014) contempla que estos sujetos, debido a su rapidez para contraatacar en respuesta a las amenazas que perciben a su alrededor, pueden ser litigantes y a menudo se vean envueltos en pleitos legales. Stone (2007) matiza estas ideas al señalar que las características de la personalidad paranoide relativas a la violencia son la rapidez para reaccionar con ira o contraatacar (sin advertir claramente la intención de los demás), guardar rencor y sospechar sin justificación ser lesionado o explotado. Los pacientes sin problemas legales relacionados con la delincuencia tuvieron puntuaciones más altas en las escalas de la personalidad narcisista, histriónica y compulsiva. 
Un resultado importante y preocupante del presente estudio es el alto número de individuos que fueron víctimas de distintos tipos de abuso: emocional (69\%), físico (42.3\%) y sexual (7\%). En este sentido, un estudio que examinaba el riesgo de adicción en adolescentes encontró una correlación positiva entre el riesgo de adicción y el haber sufrido trauma infantil (Cornellà-Font, Viñas-Poch, Juárez-López, Martín-Perpiñá y Malo-Cerrato, 2018). Además, un estudio anterior (Fernández-Montalvo et al., 2012) encontró que los pacientes adictos que exhibían comportamientos violentos eran significativamente más propensos a tener historiales de abuso. Aunque no hubo diferencias significativas en la historia de abuso en pacientes con y sin problemas relacionados con la delincuencia en este estudio, muchos pacientes en la muestra en su conjunto había experimentado abuso. Este hallazgo es consistente con otros estudios de pacientes con adicción, que han encontrado altas tasas de prevalencia de historiales de abuso entre los drogodependientes (Marshall, Fairbairn y Kerr, 2008; Fernández-Montalvo et al., 2013). Se necesitan más estudios para investigar este fenómeno y confirmar estos resultados.

El presente estudio tiene una serie de limitaciones. En primer lugar, la naturaleza exploratoria y descriptiva de este estudio significa que el papel causal específico que desempeñan las sustancias en el desarrollo de comportamientos delictivos sigue siendo desconocido. $\mathrm{Y}$ en segundo lugar, la configuración de la propia muestra es otra cuestión que debe tenerse en cuenta tanto por el número total de sujetos como por las pocas mujeres que fueron incluidas en la muestra. El posterior análisis de datos que se está realizando en la actualidad, empleando técnicas de agrupación (p.e. análisis de conglomerados) y el diseño de formulaciones de mediación y modulación permitirá conclusiones complementarias.

En resumen, el presente estudio investigó la tasa de prevalencia de comportamientos delictivos en pacientes drogadictos, así como los perfiles diferenciales de pacientes con y sin problemas legales derivados comportamientos delictivos. Los resultados del estudio solo nos permiten concluir que dada la compleja interrelación de factores y de condiciones sociales que intervienen en la génesis de ambas conductas (drogadicción y delincuencia) cualquier explicación acerca de cómo se relacionan puede ser razonable pero, en cualquier caso, será parcial. Además, es importante señalar que la relación entre drogas y delitos puede ser dinámica y variar incluso en una misma persona a lo largo del tiempo. $\mathrm{Y}$ en cuanto a la comprensión de las variables comunes que influyen en la relación droga-delincuencia consideramos que sería interesante seguir profundizando en las variables de personalidad, puesto que algunos trabajos han contribuido a reafirmar la impulsividad y la búsqueda de sensaciones como importantes factores tanto en el consumo de drogas como en la delincuencia (Carou, Romero y Luengo, 2013). Desde el punto de vista clínico, este es un objetivo importante porque la impulsividad y la búsqueda de sensaciones interfieren con el curso de la evolución terapéutica de los pacientes adictos.

\section{Conflictos de intereses}

Los autores declaran que no existen conflictos de intereses.

\section{Referencias}

Ana, E. J. S., Martino, S., Ball, S. A., Nich, C., Frankforter, T. L., y Carroll, K. M. (2008). What is usual about «treatment-asusual»? Data from two multisite effectiveness trials. Journal of Substance Abuse Treatment, 35, 369-379. https://doi. org/0.1016/j.jsat.2008.01.003

Bennett, T., y Holloway, K. (2005). The association between multiple drug misuse and crime. International Journal of Offender Therapy and Comparative Criminology, 49, 63-81. https://doi.org/10.1177/0306624X04269003

Bobes, J., González, M. P., Sáiz, P. A., y Bousoño, M. (1996). Índice europeo de severidad de la adicción: EuropASI. Versión española. Paper presented at the Actas de la IV Reunión Interregional de Psiquiatría.

Caldero, A. (2014). Estudio de los rasgos de la personalidad en población normal con el Cuestionario Salamanca. Tesis doctoral. Salamanca: Universidad de Salamanca. https://doi. org/10.14201/gredos. 127314

Cardenal, V., y Sánchez, M. P. (2007). Adaptación y baremación al español del Inventario Clínico Multiaxial de Millon-III (MCMI-III). Madrid: TEA Ediciones.

Carou, M., Romero, E. y Luengo M.A. (2013). Patrones de consumo y variables de personalidad en drogodependientes a tratamiento. Revista Española de Drogodependencias, 38 (3), 217-232.

Casares-López, M. J., González-Menéndez, A., Torres-Lobo, M., SecadesVilla, R., Fernández-Hermida, J. R., y Álvarez, M. M. (2010). Comparación del perfil psicopatológico y adictivo de dos muestras de adictos en tratamiento: en prisión y en comunidad terapéutica. International Journal of Clinical and Health Psychology, 10, 225- 243.

Contreras, L., Molina, V. y Cano, M. C. (2012). Consumo de Drogas en Adolescentes con conductas infractoras: análisis de variables psicosociales implicadas. Adicciones, 24, 31-38. https://doi.org/10.20882/adicciones. 115

Cornellà-Font, M.G., Viñas-Poch, F., Juárez-López, J.R., MartínPerpiñá, M. M. y Malo-Cerrato, S. (2018). Temperament and attachment as predictive factors for the risk of addiction to substances in adolescents. Revista de Psicopatología y Psicología Clínica, 23 (3), 179-187. https://doi.org/10.5944/ rppc.vol.23.num.3.2018.21423

Derogatis, L. R. (1992). The SCL-90-R. Baltimore: Clinical Psychometric Research. 
Esbec, E. y Echeburúa, E. (2016). Abuso de drogas y delincuencia: consideraciones para una valoración forense integral. Adicciones, 28 (1), 48-56. https://doi.org/10.20882/ adicciones. 790

Fazel, S., Bains, P., y Doll, H. (2006). Substance abuse and dependence in prisoners: a systematic review. Addiction, 101, 181-191. https://doi.org/10.1111/j.1360-0443.2006.01316.x

Felson, R. B., y Staff, J. (2010). The effects of alcohol intoxication on violent versus other offending. Criminal Justice and Behavior, 37, 1343-1360. https://doi.org/10.1177/0093854810382003

Fernandez-Montalvo, J., Landa, N., Lopez-Goni, J., y Lorea, I. (2006). Personality disorders in alcoholics: A comparative pilot study between the IPDE and the MCMI-II. Addictive Behaviors, 31, 1442- 1448. https://doi.org/10.1016/j.addbeh. 2005.09.014

Fernández-Montalvo, J., López-Goñi, J. J., Arteaga, A. y Cacho, R. (2013) Criminal profile of patients in addiction treatment. Adicciones, 25 (2), 146-155.

Fernández-Montalvo, J., Lorea, I., López-Goñi, J. J., Landa, N., y Zarzuela, A. (2003). Trastornos de personalidad en adictos a la cocaína: un estudio-piloto. Análisis y Modificación de Conducta, 29, 79-98.

Fernández-Montalvo, J., y López-Goñi, J. J. (2010). Comparison of completers and dropouts in psychological treatment for cocaine addiction. Addiction Research \& Theory, 18, 433-441. https://doi.org/10.3109/16066350903324826

Fernández-Montalvo, J., López-Goñi, J. J. y Arteaga, A. (2011). Tratamiento de agresores contra la pareja en programas de atención a drogodependientes: un reto de futuro. Adicciones, 23, 5-9. https://doi.org/10.20882/adicciones. 161

Fernández-Montalvo, J., López-Goñi, J. J., y Arteaga, A. (2012). Violent behaviours in drug addiction: Differential profiles of drug-addicted patients with and without violence problems. Journal of Interpersonal Violence, 27, 142-157. https://doi. org/10.1177/0886260511416475

Fernández-Montalvo, J., López-Goñi, J. J., Illescas, C., Landa, N., y Lorea, I. (2008). Evaluation of a therapeutic community treatment program: A long-term follow-up study in Spain. Substance Use \& Misuse, 43, 1362-1377. https://doi. org/10.1080/10826080801922231

González de Rivera, J. L. (2002). Versión española del SCL-90-R. Madrid: TEA Ediciones.

Gossop, M., Trakada, K., Stewart, D., y Witton, J. (2005). Reductions in criminal convictions after addiction treatment: 5-year followup. Drug and Alcohol Dependence, 79, 295-302. https://doi.org/10.1016/j.drugalcdep.2005.01.023

Graña, J. L., Muñoz, J. J., \& Navas, E. (2009). Normal and pathological personality characteristics in subtypes of drug addicts undergoing treatment. Personality and Individual Differences, 46, 418-423. https://doi.org/10.1016/j.paid.2008.11.011

Kokkevi, A., y Hartgers, C. (1995). EuropASI: European adaptation of a multidimensional assessment instrument for drug and alcohol dependence. European Addiction Research, 1, 208-210. https://doi.org/10.1159/000259089

Kuhns, J. B., y Clodfelter, T. A. (2009). Illicit drug-related psychopharmacological violence: The current understanding within a causal context. Aggression and Violent Behavior, 14, 69-78. https://doi.org/10.1016/j.avb.2008.11.001
Lee, C. (2007). Sex-gender differences in drug abuse: A shift in the burden of proof? Experimental and Clinical Psychopharmachology, 15, 411-417. https://doi.org/10.1037/ 1064-1297.15.5.411

López-Goñi, J. J., Fernández-Montalvo, J., y Arteaga, A. (2012). Predictive validity of the EuropAsi: Clinical diagnosis or composite scoring? Journal of Substance Abuse Treatment, 42, 392-396. https://doi.org/10.1016/j. jsat.2011.09.011

Lopez-Goñi, J., Fernandez-Montalvo, J., Menendez, J., Yudego, F., Garcia, A., y Esarte, S. (2010). Group and individual change in the treatment of drug addictions: A follow-up study in therapeutic communities. The Spanish Journal of Psychology, 13, 906-913. https://doi.org/10.1017/s1138741600002559

Marshall, B. D. L., Fairbairn, N., Li, K., Wood, E., y Kerr, T. (2008). Physical violence among a prospective cohort of injection drug users: A gender-focused approach. Drug and Alcohol Dependence, 97, 237- 246. https://doi.org/10.1016/j. drugalcdep.2008.03.028

McLellan, A. T., Luborsky, L., Woody, G. E., y O'Brien, C. P. (1980). An improved diagnostic evaluation instrument for substance abuse patients: The Addiction Severity Index. The Journal of Nervous and Mental Disease, 168, 26-33. https:// doi.org/10.1097/00005053-198001000-00006

Menard, S. W., Mihalic, S. F., y Huizinga, D. (2001). Drugs and crime revisited. Justice Quarterly, 18 (2), 269-299. https://doi. org/10.1080/07418820100094901

Ministerio del Interior. Secretaría General de Instituciones Penitenciarias (2017) Informe General de 2016. Madrid: Secretaría General de Instituciones Penitenciarias.

Millon, Th., Davis, R. y Millon, C. (1997). MCMI-III. Inventario Clínico Multiaxial de Million-III. Manual.

Ramos, V. y Garrote, G. (2009). Relación entre la conducta de consumo de sustancias y la conducta delictiva. Revista INFAD de Psicología. International Journal of Developmental and Educational Psychology, 1 (1), 647-656.

Santo-Domingo, J. (2002). El desarrollo personal del joven y el alcohol. Trastornos Adictivos, 4 (4), 223-232. https://doi. org/10.1016/s1575-0973(02)70084-6

Stone, M. H. (2007). Violencia. En Oldham, J.M., Skodol, A.E. y Bender, D.S. (Ed.). Tratado de los trastornos de la personalidad, pp. 479-494. Barcelona: Masson.

Tournier, M., Molimard, M., Cougnard, A., Abouelfath, A., Fourrier, A., y Verdoux, H. (2005). Psychiatric disorders and their comorbidity in subjects with parasuicide by intentinal drug overdose: Prevalence and gender differences. Psychiatric Research, 136, 93-100. https://doi.org/10.1016/j.psychres. 2005.06.007

Valenzuela, E. y Larroulet, P. (2010). La relación droga y delito: Una estimación de la fracción atribuible. Estudios Públicos, 119, 33-62.

Vicens, E., Tort, V., Dueñas, R.S., Muro, A., Pérez-Arnau, F., Arroyo, J.M. ...Sarda, P. (2011). The prevalence of mental disorders in Spanish prisons. Criminal Behaviour and Mental Health, 21, 321-332. https://doi.org/10.1002/cbm.815

Weltzer, S. J. (1990). The Millon Clinical Multiaxial Inventory (MCMI): A review. Journal of Personality Assessment, 55, 445-464. https://doi.org/10.1080/00223891.1990.967 
